\title{
Human Endotoxemia Activates p38 MAP Kinase and p42/44 MAP Kinase, But Not c-Jun N-terminal Kinase
}

\author{
Bernt van den Blink, ${ }^{1}$ Judith Branger, ${ }^{1,2}$ Sebastiaan Weijer, ${ }^{1}$ Sander J.H. van Deventer, ${ }^{1,3}$ \\ Tom van der Poll, ${ }^{1,2}$ and Maikel P. Peppelenbosch ${ }^{1}$ \\ ${ }^{1}$ Department of Experimental Internal Medicine, \\ ${ }^{2}$ Department of Infectious Diseases, Tropical Medicine and AIDS, \\ ${ }^{3}$ Department of Gastroenterology, Academic Medical Center, Amsterdam, The Netherlands \\ Accepted September 24, 2001
}

\begin{abstract}
Background: All three major members of the MAPK family (i.e., p38 MAPK, p42/p44 MAPK, and c-Jun N terminal kinase (JNK)) have been shown to control cellular responses to inflammation in vitro. Therefore these kinases have been designated suitable targets for anti-inflammatory therapy. However, the extent to which these kinases are actually activated during inflammation in humans in vivo has not been investigated. We employed experimental human endotoxemia, a model of systemic inflammation, to address this question.

Materials and Methods: Male volunteers were intravenously infused with $4 \mathrm{ng} / \mathrm{kg}$ bw lipopolysaccharide
\end{abstract}

(LPS). Directly before LPS infusion and up to $24 \mathrm{~h}$ thereafter, activation of p38 MAPK, p42/p44 MAPK and JNK was assessed in peripheral blood, using Western blot and in vitro kinase assays.

Results: We observed that LPS induced a strong but transient phosphorylation and activation of p38 MAPK and p42/p44 MAPK, maximal activity being reached after $1 \mathrm{hr}$ of LPS infusion. Strikingly, no JNK phosphorylation or activation was detected under these circumstances.

Conclusions: These results suggest that both inhibitors of p38 MAPK and p42/p44 MAPK but not JNK are potentially useful for anti-inflammatory therapy.

\section{Introduction}

In recent years it has emerged that members of the Mitogen Activated Protein Kinase (MAPK) family are critically involved in intracellular signal transduction, mediating cell responses to a variety of different infectious and inflammatory stimuli. In vitro, inflammatory mediators such as lipopolysaccharide (LPS), Tumor Necrosis Factor (TNF) and interleukin-1 activate p38 MAPK, c-jun $\mathrm{N}$-terminal kinase (JNK), and p42/44 MAPK (1-6). In addition, various studies have shown that pharmacological inhibitors of p38 MAPK and p42/p44 MAPK strongly influence the production of inflammatory cytokines after such stimulation (2,7-9). Hence, inhibitors of specific MAPK family members are promising new targets for anti-inflammatory therapy.

Importantly, in studies addressing the in vitro activation of MAPK family members by inflammatory agents, the concentration used of these agents often exceeds the physiological relevant concentration many fold. Hence important questions remain both with regard to the extent and the kinetics of the activation of p38 MAPK, p42/p44 MAPK, or JNK by inflammatory stimuli in humans in vivo.

Send correspondence and reprint requests to: Bernt van den Blink, Laboratory for Experimental Internal Medicine, Academic Medical Center, Room G2-130, Meibergdreef 9, 1105 AZ Amsterdam, The Netherlands. Phone: +31 20 5665910; fax: +31 20 6977192; e-mail: B.vandenBlink@amc.uva.nl.
This consideration prompted us to assay activation of the three major MAPK family members in a well-established model of systemic inflammation in human, human endotoxemia (10). Both phosphorylation and in vitro kinase activity of p38 MAPK, p42/p44 MAPK and JNK were determined in peripheral blood leukocytes. At the LPS concentration employed, clear phosphorylation and activation of both p38 MAPK and p42/p44 MAPK but not JNK was observed, contrasting the prevailing view that p38 MAPK and JNK, but not p42/p44 MAPK are the major targets for LPS signal transduction. In addition, enzyme phosphorylation was maximal $\mathrm{l} \mathrm{hr}$ after the onset of LPS treatment, which is much slower than the kinetics described for these kinases in literature $(6,11,12)$. We conclude that LPS is able to mediate MAPK family member activation in vivo, although both the nature of the kinases involved, as well as the temporal characteristics of this activation are different from that expected from existing literature data.

\section{Materials and Methods}

\section{In Vitro Stimulation of Leukocyte Cell Fractions}

Endotoxin-free reagents and plasticware were used throughout the experimental process. Blood from healthy volunteers was collected with $10 \mathrm{U} / \mathrm{ml}$ heparin (Leo Pharmaceutical Products, Weesp, the Netherlands). PBMCs were isolated by densitygradient sedimentation on Ficoll-Paque (Pharmacia, 
Uppsala, Sweden) from blood diluted 1:1 with PBS. After isolation of the PBMC fraction, the remaining non-PBMC leukocyte fraction was isolated by lysing the erythrocytes with ice-cold isotonic $\mathrm{NH}_{4} \mathrm{Cl}$ solution (155 mmol/l $\mathrm{NH}_{4} \mathrm{Cl}, 10 \mathrm{mmol} / \mathrm{l} \mathrm{KHCO}_{3}$, $0.1 \mathrm{mmol} / \mathrm{l}$ EDTA, $\mathrm{pH} \mathrm{7.4}$, and $1 \mathrm{mM}$ Pefabloc) for 15 min. PBMCs and non-PBMCs were washed twice with PBS and resuspended in RPMI (GIBCO, Grand Island, NY) supplemented with $10 \%$ human serum (from the original blood donor) in $15 \mathrm{ml}$ tubes (Becton Dickinson, Franklin Lakes, NJ). Samples, containing the PBMCs (approximately $2 \times 10^{6}$ cells each) or non-PBMCs (approximately $10 \times 10^{6}$ cells each) from $2 \mathrm{ml}$ blood, were incubated at $37^{\circ} \mathrm{C}$ for $45 \mathrm{~min}$, and then stimulated with LPS from E. coli serotype 0111:B4 (Sigma, St. Louis, MO) at the concentrations indicated. After $15 \mathrm{~min}$, ice-cold PBS was added to each tube, and cells were centrifuged at $400 \mathrm{~g}$ for $5 \mathrm{~min}$ at $4^{\circ} \mathrm{C}$. The cell pellets were lysed in $100 \mu \mathrm{l} 3 \times$ SDS-sample buffer; this mixture was briefly sonicated $(2 \times 10 \mathrm{~s})$ and boiled for $5 \mathrm{~min}$ followed by brief centrifugation and storage at $-20^{\circ} \mathrm{C}$. Aliquots of the PBMC and non-PBMC samples $(25 \mu \mathrm{l})$ were analyzed for MAPK phosphorylation using Western blot (see further).

\section{LPS Administration to Humans In Vivo}

The institutional scientific and ethics committees approved the study, and written informed consent was obtained from each subject prior to the start of the study. Eight healthy male volunteers (mean age 22, range 19-26 years) and a group of non-LPS treated volunteers $(\mathrm{n}=3)$ participated in the investigation. LPS (Escherichia coli lipopolysaccharide, lot Gl, United States Pharmacopeial Convention, Rockville, MD) was administered as a bolus intravenous injection at a dose of $4 \mathrm{ng} / \mathrm{kg}$ body weight as described earlier $(13,14)$.

\section{Sample Collection LPS Volunteers}

Venous blood was collected in heparin-containing vacutainer tubes directly before LPS administration $(\mathrm{t}=0 \mathrm{~h})$, and at 5, 15, 30, and $60 \mathrm{~min}$ and 4, 8, and $24 \mathrm{~h}$ thereafter. Erythrocytes from $4.5 \mathrm{ml}$ aliquots were lysed by adding $40 \mathrm{ml}$ ice-cold isotonic $\mathrm{NH}_{4} \mathrm{Cl}$ solution for $30 \mathrm{~min}$. The remaining leukocytes were centrifuged for $5 \mathrm{~min}, 400 \mathrm{~g}$, at $4^{\circ} \mathrm{C}$, washed twice with ice cold PBS, and resuspended in $400 \mu \mathrm{l}$ of PBS. $200 \mu \mathrm{l}$ of the cell suspension were added to $125 \mu \mathrm{l}$ of $3 \times$ SDS-sample buffer, briefly sonicated $(2 \times 10 \mathrm{~s})$ and boiled for $5 \mathrm{~min}$ followed by brief centrifugation and storage at $-20^{\circ} \mathrm{C}$. To the remaining cell suspension, intended for the kinase assay, $800 \mu \mathrm{l}$ ice-cold cell lysis buffer $(20 \mathrm{mM}$ Tris (pH 7.5), $150 \mathrm{mM} \mathrm{NaCl,} \mathrm{ImM} \mathrm{EDTA,} \mathrm{ImM} \mathrm{EGTA,}$ $1 \%$ Triton, $2.5 \mathrm{mM}$ sodium pyrophosphate, $1 \mathrm{mM} \beta$ glycerolphosphate, $1 \mathrm{mM} \mathrm{Na} \mathrm{VO}_{4}, 1 \mu \mathrm{g} / \mathrm{ml}$ Leupeptin and ImM Pefabloc) were added. Samples were sonicated $4 \times 5 \mathrm{~s}$ on ice and spun at $7000 \mathrm{~g}$ for $10 \mathrm{~min}$ at $4^{\circ} \mathrm{C}$. Protein content in the clear supernatant was determined using a bicinchoninic acid (BCA) protein assay kit (Pierce, Rockford, IL), using BSA as the standard, and the supernatant was stored at $-80^{\circ} \mathrm{C}$.

\section{Western Blotting and Kinase Assays}

Analysis of MAPK phosphorylation was performed using Western blotting and polyclonal rabbit antibodies against phosphorylated $\left(\mathrm{Thr}^{202} / \mathrm{Tyr}^{204}\right) \mathrm{p} 44 / 42$ MAPK, phosphorylated $\left(\mathrm{Thr}^{183} / \mathrm{Tyr}^{185}\right) \mathrm{JNK}$, and phosphorylated $\left(\mathrm{Thr}^{180} / \mathrm{Tyr}^{182}\right.$ ) p38 MAPK (Cell Signaling, Beverly, MA). MAPK enzymatic activity was assayed using commercial in vitro kinase assays (Cell Signaling, Beverly, MA) according to the manufacturers protocol as described $(7,15,16)$. Extracts from UV-treated 293 cells served as a positive control for JNK (Cell Signaling, Beverly, MA).

\section{Analysis of MAPK Phosphorylation}

Phosphorylation of MAPKs was measured by Western blot. Antibody binding was quantified using image analysis software (EFM Software, Rotterdam, the Netherlands) and measurements were corrected for the amount of protein loaded to allow quantitative comparison between blood samples, which may contain different amounts of leukocyte protein as a consequence of the LPS challenge, and were compared to a reference sample (set on a 100 arbitrary units) as to allow investigation into the variation in MAPK phosphorylation between the subjects.

\section{Results}

Activation of MAPK Family Members by Endotoxin In Vitro.

In order to investigate the effects of endotoxin on the phosphorylation status of MAPK family members, peripheral blood was obtained from volunteers. Subsequently, the PBMC fraction (mainly monocytes and lymphocytes) was separated from the leukocyte non-PBMC fraction (mainly granulocytes). Both fractions were stimulated with LPS as to obtain insight into possible differences between the different cell types of the blood with respect to their reaction to endotoxin. As evident from Fig. 1, both the PBMC fraction as well as the non-PBMC fraction reacted to LPS with enhanced phosphorylation of both p38 MAPK, p42/p44 MAPK and JNK, especially at LPS concentrations in excess of $1 \mathrm{ng} / \mathrm{ml}$. We concluded that ex vivo all three MAPK family members are a target for endotoxin signal transduction and that-ex vivo-no significant differences between blood cell fractions exist.

\section{The Phosphorylation Status of MAPK Family Members is Not Subject to a Circadian Rhythm}

In order to investigate the effects of endotoxin on the phosphorylation status of MAPK members throughout a $24 \mathrm{hr}$ study period, it is essential that in unchallenged volunteers this phosphorylation status is 


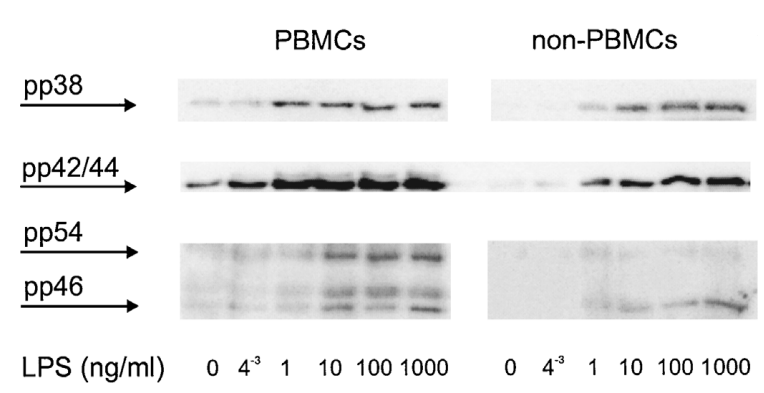

Fig. 1. LPS activates p38 MAPK, p42/44 MAPK and JNK in PBMCs and non-PBMCs in vitro. PBMCs and non-PBMCs were isolated from whole blood, incubated for $45 \mathrm{~min}$ at $37^{\circ} \mathrm{C}$, and stimulated with LPS for $15 \mathrm{~min}$ at the concentrations indicated. The leukocyte fraction (PBMC or non-PBMC) was loaded on one gel, in a quantity relative to their proportion in whole blood as to allow comparison of their relative contribution to MAPK activation in the total leukocyte fraction. MAPK activation was assessed on Western blot using phosphospecific antibodies for p38 MAPK (pp38, A), p42/44 MAPK (pp42/44, B), and JNK (pp46/pp54, C). Western blots shown are representative of three experiments.

not subject to a circadian rhythm. Hence blood was obtained from 3 volunteers at different time points and MAPK phosphorylation was investigated using Western blot analysis and phosphospecific antibodies. It appeared that p38 MAPK and p42/p44 MAPK phosphorylation in leukocytes of non LPS-treated volunteers is not subject to variation throughout the day (Fig. 2). Strikingly, no basal JNK phosphorylation was observed, although such phosphorylation was easily discernable in UV-treated human cancer cells, and thus the absence of basal JNK activation is not due to a detection problem. Hence it is possible to study the effects of LPS on this phosphorylation over a multi-hour time course.

\section{Endotoxemia Induces Transient Phosphorylation of p 38 MAPK and p42/p44 MAPK}

Subsequently, we studied the effects of human endotoxemia on the phosphorylation of these MAPKs. Infusion of LPS caused mild fever and other typical

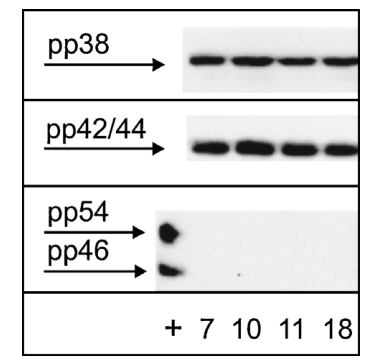

Fig. 2. No variation in MAPK phosphorylation throughout the day. Peripheral blood leukocytes from non LPS-treated volunteers, obtained at 7:00 h, 10:00 h, 11:00 h and 18:00 h (7, 10,11 , and 18 in figure), were analyzed for p38 MAPK (pp38), p42/44 MAPK (pp42/44), and JNK (pp46/pp54) phosphorylation status. (Representative examples out of three.) UV light treated 293 cells served as a positive control for JNK phosphorylation $(+)$.

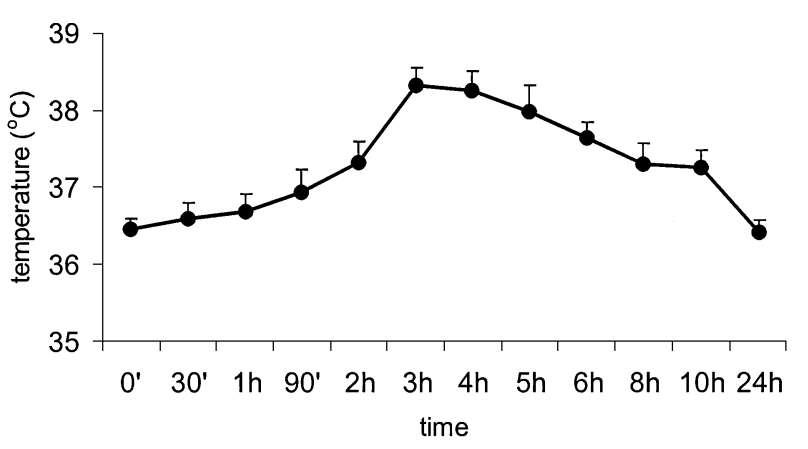

Fig. 3. Endotoxemia induces transient fever in humans. Eight healthy male volunteers were intravenously infused with a bolus of LPS (4 ng/kg bw). Body temperature was measured just before LPS infusion $\left(0^{\prime}\right)$, and $1,1^{1 / 2}\left(90^{\prime}\right), 2,4,6,8,10$ and $24 \mathrm{~h}$ after LPS infusion. Results are given as mean $\left({ }^{\circ} \mathrm{C}\right) \pm$ SEM.

symptoms as is well described for human experimental endotoxemia $(10,13,14$; Fig. 3). Importantly, strong phosphorylation of p38 MAPK and p42/p44 MAPK was observed (Fig. 4A and 5A). Using digital imaging software, the relative phosphorylation levels at different time points were determined and an aggregate curve of the combined volunteers was constructed (see experimental procedures) revealing
A

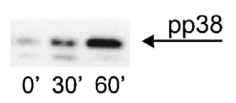

B

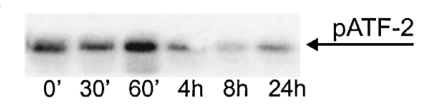

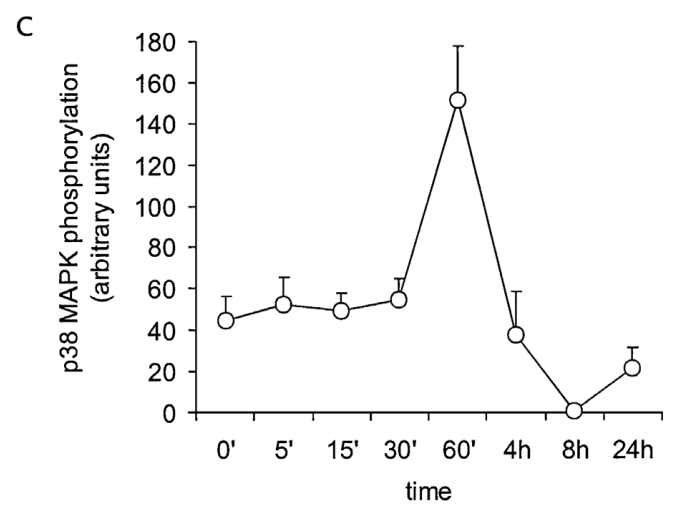

Fig. 4. Human endotoxemia activates p38 MAPK. Peripheral blood leukocytes from LPS-treated volunteers were obtained just before LPS infusion $\left(0^{\prime}\right)$, and $5,15,30$, and $60 \mathrm{~min}$, and 4,8 and $24 \mathrm{~h}$ after LPS infusion. p38 MAPK phosphorylation and enzymatic activity was then assessed at various time points. All Western blots shown are representative of at least three experiments. (A) p38 MAPK phosphorylation was determined using antibodies against phosphorylated p38 MAPK (pp38). (B) p38 MAPK enzymatic activity was determined by measuring phosphorylation of ATF-2 (pATF-2) in an in vitro kinase assay, using immunoprecipitated phosphorylated p38 MAPK from leukocyte lysates. (C) Kinetic analysis of p38 MAPK phosphorylation in leukocytes samples from 8 LPStreated volunteers. Results (mean \pm SEM) were obtained by image analysis of Western blotting with phosphospecific antibodies and expressed relative to a reference sample, which was set on a 100 arbitrary units. 
A

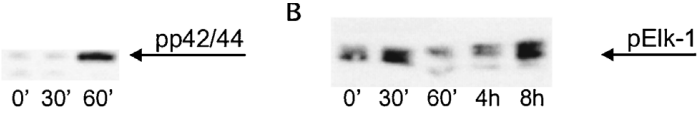

$\mathrm{C}$

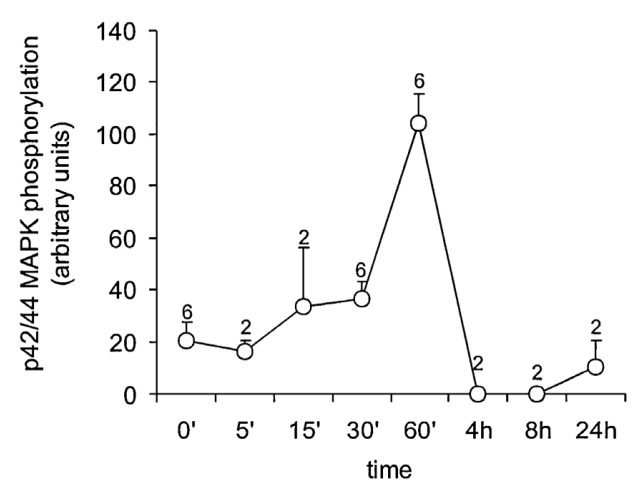

Fig. 5. Human endotoxemia activates p42/44 MAPK. Peripheral blood leukocytes from LPS-treated volunteers were obtained just before LPS infusion $\left(0^{\prime}\right)$, and $5,15,30$, and $60 \mathrm{~min}$, and 4,8 and $24 \mathrm{~h}$ after LPS infusion. P42/44 MAPK phosphorylation and enzymatic activity was then assessed at various time points. All Western blots shown are representative of at least three experiments. (A) p42/44 MAPK phosphorylation was determined using antibodies against phosphorylated p42/44 MAPK (pp42/44). (B) p42/44 MAPK enzymatic activity was determined by measuring phosphorylation of Elk-1 (pElk-1) in an in vitro kinase assay, using immunoprecipitated phosphorylated p42/44 MAPK from leukocyte lysates. (C) Kinetic analysis of p42/44 MAPK phosphorylation in leukocytes samples from LPS-treated volunteers (number of samples analyzed as indicate in the figure). Results (mean \pm SEM) were obtained by image analysis of Western blotting with phosphospecific antibodies and expressed relative to a reference sample, which was set on a 100 arbitrary units.

the temporal characteristics of this phosphorylation. On average maximal phosphorylation was observed $60 \mathrm{~min}$ after LPS infusion, followed by a striking dephosphorylation of both kinases (Fig. 4C and 5C). Importantly, however, in vivo LPS challenge was not accompanied by increased phosphorylation of either p54 JNK or p46 JNK (Fig. 6A), whereas
A

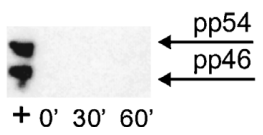

B

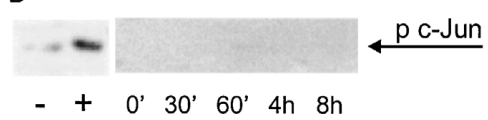

Fig. 6. Human endotoxemia does not activate JNK. Peripheral blood leukocytes from LPS-treated volunteers were obtained just before LPS infusion $\left(0^{\prime}\right)$, and $5,15,30$, and $60 \mathrm{~min}$, and 4,8 and $24 \mathrm{~h}$ after LPS infusion. JNK phosphorylation and enzymatic activity was then assessed at various time points. All Western blots shown are representative of at least three experiments. (A) JNK phosphorylation was determined using antibodies against phosphorylated JNK (pp46/pp54), UV light treated 293 cells served as a positive control for JNK phosphorylation (+). (B) JNK enzymatic activity was determined by measuring phosphorylation of c-Jun (p c-Jun) in an in vitro kinase assay, using immunoprecipitated phosphorylated JNK from leukocyte lysates, HeLa cells treated with osmoshock or left untreated served as a positive and negative control $(+,-)$. in vitro this phosphorylation was easily detectable. Thus human endotoxemia provokes strong but transient phosphorylation of both p38 MAPK and p42/p44 MAPK, but JNK phosphorylation is not detected.

\section{LPS Infusion Enhances p38 MAPK and p42/p44 MAP Enzymatic Activity in Peripheral Blood Leukocytes}

To confirm that enhanced phosphorylation of MAPKs after in vivo LPS challenge is accompanied by enhanced enzymatic activity of these enzymes, MAPKs were immunoprecipitated from peripheral blood lysates. As evident from Fig. 4B, phosphorylation of p38 MAPK was accompanied by increased enzymatic activity. Also p42/p44 MAPK showed enhanced enzymatic activity after LPS infusion, but the kinetics did not completely correspond to enzyme phosphorylation, suggesting that other intracellular regulatory events, directing activity of p42/p44 MAPK, are present in vivo as well (Fig. 5B). Strikingly, neither phosphorylation nor activation of JNK was observed in the white cell fraction of our LPS volunteers (Fig. $6 \mathrm{~A}, \mathrm{~B})$. These results confirm that human endotoxemia results in phosphorylation and activation of $\mathrm{p} 38$ MAPK and p42/p44 MAPK. Hence these results form the first formal proof of the activation of MAPK family members in an inflammatory setting during human inflammation.

\section{Discussion}

A large body of scientific research has led to the realization that the MAPK family is a principal regulator of cell physiology. Activation of p38 MAPK, p42/p44 MAPK and JNK in response to a variety of inflammatory agents has been described and these MAPKs control many cellular responses to inflammation. As a consequence, inhibitors of these kinases have been proposed as anti-inflammatory therapy. Importantly, however, actual activation of these kinases during human inflammation has not been verified and hence we set out to investigate the effects of human endotoxemia on MAPKs. To this end we investigated endotoxin responses in different blood cell fractions ex vivo and compared these to the effects observed in the human endotoxemia model. This well-established model involves rapid systemic inflammation and simultaneous activation of many inflammatory cells, and we argued that under these circumstances MAPK activation should be readily detectable.

Indeed, endotoxemia induced both phosphorylation and enzymatic activation of p38 MAPK and p42/44 MAPK in peripheral blood leukocytes, suggesting that these kinases are important in mediating LPS effects in vivo. Hence, it should be interesting to investigate whether pharmacological inhibition of such kinases will result in reduced fever and suppression of the other typical inflammatory symptoms observed in the human endotoxemia model. Interestingly, LPS induced activation of 
MAPKs was followed by dephosphorylation of both p38 MAPK and p42/44 MAPK. In vitro, LPS pretreatment inhibits LPS-induced p38 MAPK and p42/44 MAPK activation, and this phenomenon is suggested to be involved in LPS tolerance $(17,18)$. The mechanism underlying LPS tolerance is thought to reflect a protective cellular reprogramming against shock due to hyperreactive inflammatory cells, but on the other hand may render septic patients vulnerable to secondary infections. Our data support the notion that in vivo exposure to LPS eventually inhibits MAPK activation. This MAPK inhibition possibly induces a refractory state in MAPK activation and may be involved in cellular hyporesponsiveness during shock, but obviously further experimental works is warranted. LPS-induced phosphorylation and activation of p38 MAPK and p42/ p44 MAPK was fairly slow compared to in vitro studies $(6,11,12)$, even allowing for the time necessary to obtain homogenous plasma distribution of LPS. This may be due to the fact that the final LPS concentration reached in endotoxemic volunteers is relatively low as compared to most in vitro studies. Alternatively, LPS presentation to cells in vivo is far more complex then in vitro, involving competing influences of LPS binding protein and bactericidal/ permeability-increasing protein (19), which may explain kinetic differences. Nevertheless, these experiments identify these kinases as relevant targets for LPS in vivo and suggest that both p38 MAPK and $\mathrm{p} 42 / \mathrm{p} 44$ MAPK are of interest with regard to therapeutical relevance in systemic inflammation. Experiments addressing the effects of such inhibitors are currently in progress.

In contrast to p38 MAPK and p42/44 MAPK, JNK activation could not be detected during human endotoxemia, while in vitro these kinases are often simultaneously stimulated by LPS and other stimuli $(12,20$, 21). JNK phosphorylation is in general more difficult to detect when compared to the phosphorylation of other MAPK members, thus the possibility exists that JNK activation in vivo is below our detection limit. However, ex vivo JNK phosphorylation was easily detectable and also positive controls were unambiguous. The absence of LPS-dependent JNK activity in vivo may also be a consequence of the low concentration of LPS reached in the volunteers, or reflect differences in cellular physiology occurring in circulating leukocytes compared to ex-vivo stimulated cells.

MAPK activation was assayed in total leukocyte samples from peripheral blood, as preliminary experiments suggested that more elaborate separations into different cell fractions results in hyperphosphorylation and activation (unpublished observations). Earlier reports, using ex vivo stimulated neutrophils, have explicitly not observed LPS-induced JNK activation in neutrophils (22), while LPS-induced JNK activation in monocytes is well described (23). This raises the concern whether the relative large proportion of neutrophils in the peripheral blood of our volunteers might obscure LPS induced JNK activation in other leukocyte cell fractions such as PBMCs. However, in our ex vivo experiments we separated the PBMC cell fraction from the non-PBMC fraction (ie., mainly neutrophils). The amount of leukocyte fractions (PBMC or non-PBMC) loaded on a gel was relative to their proportion in whole blood as to allow comparison of their relative contribution to the measured MAPK activation in the total leukocyte samples assayed from the endotoxemia volunteers. In the nonPBMC fraction we did find LPS-induced JNK activation, suggesting that LPS can activate JNK in neutrophils, contrasting an earlier report (22). Recently, however, it was reported that TNF induces JNK activation in neutrophils in an adhesion dependent fashion (24). It is very well conceivable that LPS induced JNK activation, similarly to TNF, is adhesion dependent in neutrophils. The non-adherent peripheral blood neutrophils, obtained from endotoxemic volunteers, may thus lack adhesion-dependent JNK activation in contrast to the neutrophils present in the non-PBMC fraction that were stimulated in a fashion allowing adhesion (see material and methods) and this may possibly explain the difference between our in vivo and in vitro results. Experiments in which the phosphorylation status of MAPK family members is investigated using intracellular FACS analysis may in future help to address the problem of differential MAPK activation in different cell types.

Nevertheless, the absence of LPS-induced JNK activity in vivo, relative to the clear p38 MAPK and p42/44 MAPK activation, suggests that JNK may not be an ideal target for anti-inflammatory therapy.

\section{References}

1. Minden A, Lin A, McMahon M, et al. (1994) Differential activation of ERK and JNK mitogen-activated protein kinases by Raf-1 and MEKK. Science 266: 1719-1723.

2. Lee JC, Laydon JT, McDonnell PC, et al. (1994) A protein kinase involved in the regulation of inflammatory cytokine biosynthesis. Nature 372: 739-746.

3. Coso OA, Chiariello M, Yu JC, et al. (1995) The small GTPbinding proteins Racl and Cdc42 regulate the activity of the JNK/SAPK signaling pathway. Cell 81: 1137-1146.

4. Liu MK, Herrera-Velit P, Brownsey RW, Reiner NE. (1994) CD 14-dependent activation of protein kinase $C$ and mitogenactivated protein kinases (p42 and p44) in human monocytes treated with bacterial lipopolysaccharide. J. Immunol. 153: 2642-2652.

5. Gupta S, Barrett T, Whitmarsh AJ, et al. (1996) Selective interaction of JNK protein kinase isoforms with transcription factors. $E M B O . J$. 15: 2760-2770.

6. Hambleton J, Weinstein SL, Lem L, DeFranco AL. (1996) Activation of c-Jun N-terminal kinase in bacterial lipopolysaccharide-stimulated macrophages. Proc. Natl. Acad. Sci. USA 93: 2774-2778.

7. van den Blink B, Juffermans NP, ten Hove T, et al. (2001) p38 mitogen-activated protein kinase inhibition increases cytokine release by macrophages in vitro and during infection in vivo. J. Immunol. 166: 582-587.

8. Beyaert R, Cuenda A, Vanden Berghe W, et al. (1996) The p38/RK mitogen-activated protein kinase pathway regulates interleukin-6 synthesis response to tumor necrosis factor. ЕМВО. J. 15: 1914-1923. 
9. Scherle PA, Jones EA, Favata MF, et al. (1998) Inhibition of MAP kinase kinase prevents cytokine and prostaglandin E2 production in lipopolysaccharide-stimulated monocytes. $J$. Immunol. 161: 5681-5686.

10. van der Poll T, van Deventer SJH. (1999) Endotoxemia in healthy subjects as a human model of inflammation. In: Cohen J, Marshall J (eds.) The Immune Response in the Critically Ill, Springer-Verlag, USA/Germany, pg. 335-357.

11. Carter AB, Monick MM, Hunninghake GW. (1999) Both Erk and p38 kinases are necessary for cytokine gene transcription. Am. J. Respir. Cell. Mol. Biol. 20: 75 1-758.

12. Zhu W, Downey JS, Gu J, et al. (2000) Regulation of TNF expression by multiple mitogen-activated protein kinase pathways. J. Immunol. 164: 6349-6358.

13. van der Poll T, Calvano SE, Kumar A, et al. (1995) Endotoxin induces downregulation of tumor necrosis factor receptors on circulating monocytes and granulocytes in humans. Blood 86: 2754-2759.

14. van der Poll T, Coyle SM, Barbosa K, et al. (1996) Epinephrine inhibits tumor necrosis factor-alpha and potentiates interleukin 10 production during human endotoxemia. J. Clin. Invest. 97: 713-719.

15. Versteeg HH, Nijhuis E, van den Brink GR, et al. (2000) A new phosphospecific cell-based ELISA for p42/p44 mitogenactivated protein kinase (MAPK), p38 MAPK, protein kinase $\mathrm{B}$ and cAMP-response-element-binding protein. Biochem. $J$. 350 Pt 3: 717-722.

16. Yokoi T, Ohmichi M, Tasaka K, et al. (2000) Activation of the luteinizing hormone beta promoter by gonadotropinreleasing hormone requires c-Jun $\mathrm{NH} 2$-terminal protein kinase. J. Biol. Chem. 275: 21639-21647.
17. Medvedev AE, Kopydlowski KM, Vogel SN. (2000) Inhibition of lipopolysaccharide-induced signal transduction in endotoxin-tolerized mouse macrophages: dysregulation of cytokine, chemokine, and toll-like receptor 2 and 4 gene expression. J. Immunol. 164: 5564-5574.

18. Mendez C, Kramer AA, Salhab KF, et al. (1999) Tolerance to shock: an exploration of mechanism. Ann Surg 229: 843-849; discussion 849-850.

19. Jin H, Yang R, Marsters S, et al. (1995) Protection against endotoxic shock by bactericidal/permeability-increasing protein in rats. J. Clin. Invest. 95: 1947-1952.

20. De Cesaris P, Starace D, Riccioli A, et al. (1998) Tumor necrosis factor-alpha induces interleukin-6 production and integrin ligand expression by distinct transduction pathways. $J$. Biol. Chem. 273: 7566-7571.

21. Chan ED, Winston BW, Uh ST, et al. (1999) Evaluation of the role of mitogen-activated protein kinases in the expression of inducible nitric oxide synthase by IFN-gamma and TNFalpha in mouse macrophages. J. Immunol. 162: 415-422.

22. Nick JA, Avdi NJ, Gerwins P, et al. (1996) Activation of a p38 mitogen-activated protein kinase in human neutrophils by lipopolysaccharide. J. Immunol. 156: 4867-4875.

23. Hambleton J, Weinstein SL, Lem L, DeFranco AL. (1996) Activation of c-Jun N-terminal kinase in bacterial lipopolysaccharide-stimulated macrophages. Proc. Natl. Acad. Sci. USA 93: 2774-2778.

24. Avdi NJ, Nick JA, Whitlock BB, et al. (2001) Tumor necrosis factor-alpha activation of the c-Jun $\mathrm{N}$-terminal kinase pathway in human neutrophils. Integrin involvement in a pathway leading from cytoplasmic tyrosine kinases apoptosis. $J$. Biol. Chem. 276: 2189-2199. 